\title{
Surgical Outcome in Cubital Tunnel Syndrome
}

\author{
Oertel J* and Keiner D
}

Department of Neurosurgery, University of Saarland

${ }^{*}$ Corresponding author: Oertel J, Department of Neurosurgery, University of Saarland, Kirrbergerstrasse buildings 90.5, 66424 Homburg Saar, Fax: 49-6841-16-24480, Tel: 49-6841-16-24400, E-mail: oertelj@ freenet.de

\author{
Citation: Oertel J, Keiner D (2015) Surgical Outcome in Cubital Tunnel Syndrome. J Surg Oper Care 1(1): \\ 104. doi: $10.15744 / 2455-7617.1 .104$
}

Received Date: May 24, 2015 Accepted Date: October 30, 2015 Published Date: November 03, 2015

\begin{abstract}
'Cubital tunnel syndrome represents the second most common nerve compression syndrome. While mild cases have been well treated conservatively at first hand, surgical therapy should be considered early on, because atrophy of the intrinsic muscles is often irreversible [1]. Although the complication rate of surgical therapy is low and a favourable outcome is reported commonly in the present literature, the overall results are moderate compared to surgical results in other arm- or hand-associated diseases. In the past decades, various different techniques for the treatment of the cubital tunnel syndrome have been applied including medial epicondylectomy, subcutaneous, submuscular or intramuscular anterior transposition, simple decompression, and endoscopicassisted decompression [2,3]. However, no surgical technique has been proven to be significantly superior to the other techniques so far [1].
\end{abstract}

Based on systematic reviews and meta-analyses [1,4,5], it has been concluded that simple decompression has an equivalent clinical outcome and a lower incidence of complications compared with anterior transposition. Presently, simple decompression with complete opening of the cubital tunnel retinaculum has been suggested as the therapy of choice [4-6]. In the past years, shear endoscopic and endoscopic-assisted decompression of the ulnar nerve at the elbow have been performed in several institutions with good results, but no larger prospective-randomized studies have been published so far [7-9].

Besides meticulous physical examination and neurography, which are of utmost importance for the successful treatment of cubital tunnel syndrome, ultrasonography and magnetic resonance imaging have been used more frequently for the diagnostic evaluation and for postoperative outcome measurement in the past years.

Although the severity of sensory and motor symptoms is commonly classified into different grading systems (i.e. Dellon classification, McGowan grading classification), measurement of postoperative outcome in terms of patient's satisfaction, overall hand function in daily life and quality of life is difficult.

In the manuscript entitled "Surgical outcome of cubital tunnel syndrome using the MHQ und DASH: a retrospective patientreported assessment", Sorg et al. present their results of a retrospective outcome measurement using the Disabilities of the Arm, Shoulder and Hand outcome measure (DASH) and German Michigan Hand Questionaire (MHQ) in 19 out of 41 patients, who were treated surgically for cubital tunnel syndrome in their institution between 2006 and 2010. Emphasis was placed on the evaluation of the quality of life following subcutaneous or submuscular transposition of the ulnar nerve using detailed questionnaires. The aim of the study was not to prove a superiority of one surgical technique against another, but to present a tool that may be helpful to evaluate the postoperative surgical success. In the clinical daily routine, the obtained data provides detailed information of a single patient and the individual postoperative course. Therefore, the questionnaires might be as well useful as an additional tool for the evaluation of different surgical techniques.

However, there is one important point that is missing in the present work. Only very few details are given regarding the preoperative findings such as the duration of symptoms or the severity of neurological findings. It would be very useful to add the preoperative findings in form of a "classical" grading system i.e. McGowan or otherwise, because the postoperative outcome often depends on the preoperative course. In many cases, patients with severe preoperative neurological deficits are very satisfied with a postsurgical improvement even if some clinical signs remain, whereas patients with moderate preoperative symptoms are often only satisfied in case of complete improvement of their symptoms. Due to the retrospective design of the present study it is impossible to add the information in form of DASH and MHQ, but in the future it would be interesting to add these questionnaires in prospective studies regarding the surgical technique in cubital tunnel syndrome.

Nevertheless we congratulate the authors for their new approach to evaluate patient's satisfaction and await further publications which include more preoperative data and allow a diversification of the various surgical techniques. 


\section{References}

1. Assmus H, Antoniadis G, Bischoff C (2015) Carpal and cubital tunnel and other, rarer nerve compression syndromes. Dtsch Arztebl Int 112: $14-25$.

2. Tang DT, Barbour JR, Davidge KM, Yee A, Mackinnon SE (2015) Nerve Entrapment: Update. Plast Reconstr Surg 135: 199-215e.

3. Bartels RMH (2001) History of the surgical treatment of ulnar nerve compression at the elbow. Neurosurgery 49: 391-9.

4. Chen H, Ou S, Liu G, Fei J, Zhao G, et al. (2014) Clinical efficacy of simple decompression versus anterior transposition of the ulnar nerve for the treatment of cubital tunnel syndrome: A meta-analysis. Clinical Neurology and Neurosurgery 126: 150-5.

5. Kamat AS, Jay SM, Benoiton LA, Correia JA, Woon K (2014) Comparative outcomes of ulnar nerve transposition versus neurolysis in patients with entrapment neuropathy at the cubital tunnel: a 20-year analysis. Acta Neurochir (Wien) 156: 153-7.

6. Assmus H, Antoniadis G, Bischoff C, Hoffmann R, Martini AK, et al. (2011) Cubital tunnel syndrome - a review and management guidelines. Cent Eur Neurosurg 72: 90-8.

7. Ahcan U, Zorman P (2007) Endoscopic decompression of the ulnar nerve at the elbow. J Hand Surg Am 32: 1171-6.

8. Oertel J, Keiner D, Gaab MR (2010) Endoscopic decompression of the ulnar nerve at the elbow. Neurosurgery 66: 817-24.

9. Hoffmann R, Lubahn J (2013) Endoscopic cubital tunnel release using the Hoffmann technique. J Hand Surg Am 38: $1234-9$.

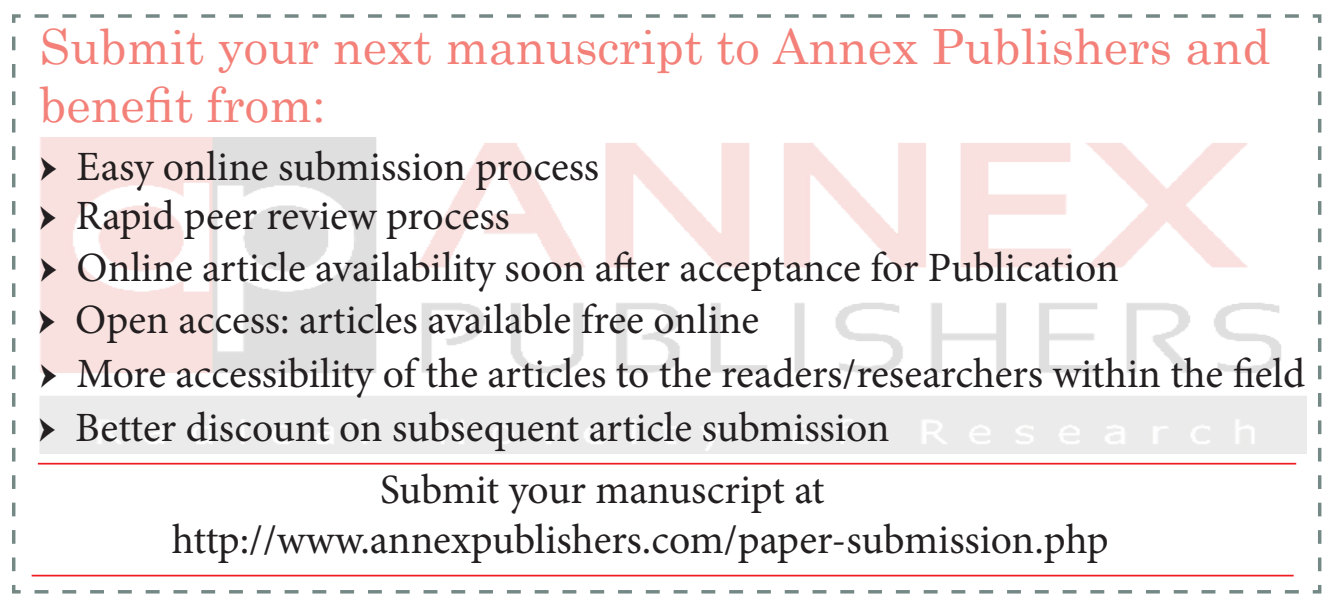

